\title{
Treatment Efficiency and Problems of Local Control in Localized Bladder/Prostate Rhabdomyosarcoma
}

\author{
Hanafy Hafez ${ }^{1}$, Enas EI Nadi ${ }^{2}$, Alaa Younes ${ }^{3}$, Gehad Ahmed $^{4}$, Mohamed Saad Zaghloul, \\ Hala Taha $^{6}$, Rania Labib ${ }^{7}$, Sayed Abdelhamid Fadel ${ }^{1}$, Soha Ahmed ${ }^{8}$ \\ ${ }^{1}$ Pediatric Oncology, National Cancer Institute (NCI), Cairo University, Cairo, Egypt \\ ${ }^{2}$ Pediatric Oncology Department, Beni Suef University, Beni Suef, Egypt \\ ${ }^{3}$ Surgical Oncology, National Cancer Institute, Cairo University, Cairo, Egypt \\ ${ }^{4}$ Surgery Department, Faculty of Medicine, Helwan University, Helwan, Egypt \\ ${ }^{5}$ Radiation Oncology, National Cancer Institute (NCI), Cairo University, Cairo, Egypt \\ ${ }^{6}$ Surgical Pathology, National Cancer Institute (NCI), Cairo University, Cairo, Egypt \\ ${ }^{7}$ Clinical Research Department, Children Cancer Hospital Egypt, Cairo, Egypt \\ ${ }^{8}$ Department of Clinical Oncology, Aswan University, CCHE, Cairo, Egypt
}

\section{Email address:}

Hafez@57357.org(H. Hafez), inas.mohsen@57357.org (E. El Nadi), alaa.younes@57357.org (A. Younes), gehad.taha@gmail.com (G.Ahmed),mszagh@yahoo.com(M. S.Zaghloul),hala.taha@57357.com(H. Taha), rania.labib@57357.org(R. Labib), sayedmarwan91@yahoo.com(S. A. Fadel)

\section{To cite this article:}

Hanafy Hafez, Enas El Nadi, Alaa Younes, Gehad Ahmed, Mohamed Saad Zaghloul, Hala Taha, Rania Labib, Sayed Abdelhamid Fadel, Soha Ahmed. Treatment Efficiency and Problems of Local Control in Localized Bladder/Prostate Rhabdomyosarcoma. Cancer Research Journal. Vol. 7, No. 2, 2019, pp. 33-38. doi: 10.11648/j.crj.20190702.11

Received: February 19, 2019; Accepted: March 22, 2019; Published: April 18, 2019

\begin{abstract}
Objectives: To assess the treatment efficiency, outcome and factors affecting the local control of localized bladder/prostate RMS. Patients and methods: Retrospective analysis of 54 patients with localized bladder/prostate RMS treated at Children Cancer Hospital, Egypt between August 2007 and Jan 2017. All patients were treated according to Intergroup Rhabdomyosarcoma Study (IRS -V) and subsequent Children's Oncology Group COG guidelines. Results: The median age at diagnosis was 3.28 years (range 0.4-13.6). Fifty-one patients (94\%) underwent initial biopsy. Complete surgical resection (primary or delayed) was performed in 7 patients (13\%). Local control started before/at week 12 in 29 patients (61.7\%). Local control methods were: Radiotherapy in 43 patients $(79.2 \%)$, radiotherapy with surgery in 4 patients $(9 \%)$, surgery in 1 patient and four patients did not receive local control. With a median follow up of 38.12 months, the 5-year failure-free survival (FFS) and overall (OS) of the whole patients were $60.4 \pm 14.5 \%$ and $75.4 \pm 14.1 \%$ respectively. A better 5-year FFS was experienced by those who had early local control $(79.2 \pm 17 \%$ vs. $43.8 \pm 25 \% \mathrm{p}=0.005)$. Conclusions: Timing of local control and local radiotherapy is crucial and shouldn't be delayed waiting for further response to the systemic chemotherapy.
\end{abstract}

Keywords: RMS, Bladder/Prostate, Local Control

\section{Introduction}

Rhabdomyosarcoma accounts for $3.5 \%$ of cancer among children up to 14 years of age representing the third most common extra cranial solid tumor of childhood after neuroblastoma and wilms tumor. The three currently recognized modalities in treatment of childhood sarcomas are surgical resection (if feasible), systemic chemotherapy (for primary cytoreduction and eradication of gross and micro metastases) and radiation therapy for control of residual bulk or microscopic tumor. Bladder/prostate Rhabdomyosarcoma is the second most common primary site representing approximately $10-15 \%$ of all RMS cases, with a predilection to the trigone of the bladder [1-3]. The main histological subtypes are embryonal and alveolar [4]. Embryonal histology accounts for $90 \%$ of genitourinary RMS, and has a more favorable prognosis (82\% 5-year EFS) than alveolar 
pathology (65\% 5-year EFS) [5]. The common presenting symptoms are gross hematuria, difficulty in voiding, urinary retention, and abdominal mass $[2,6]$. A multidisciplinary treatment approach including surgery, radiotherapy, and chemotherapy is used in these patients aiming at better survival and as much as possible function preservation [7-9]. Previously published trials reported 5-year survival in the range of 71 and $82 \%$ together with improved rates of bladder conservation $[7,9,10]$.

The local treatment of (BP)-RMS is challenging and up till now it remains unclear, which treatment strategy is optimal for local control. Radiotherapy might help reduce tumor volumes and therefore might avoid radical surgery at the expense of high post-treatment morbidity [10]. Bladder/prostate is considered an unfavorable primary site and most cases are not suitable for primary surgery (IRS-III). Therefore, the vast majority of BP-RMS is attributed to standard or high-risk groups $[11,12]$. The current controversies in managing $\mathrm{B} / \mathrm{P}$ RMS lie in the timing, order of surgical or radiotherapy, treatment of residual disease after initial chemotherapy and modality of radiotherapy. The Objectives of this study were to assess the treatment efficiency, outcome and factors affecting the local control of localized bladder/prostate RMS.

\section{Patients and Methods}

Children and adolescents younger than 18 years, with newly diagnosed localized bladder/prostate RMS, that were treated at Children Cancer Hospital Egypt (CCHE) from August 2007 to Jan 2017were included. After the institutional review board (IRB) approval, Data were collected. Including patient's demographics (age and sex), tumor site, size, lymph node involvement, staging, pathology and methods \& timing of local control. Initial diagnosis was made by Ultrasonography, and was followed by MRI of the abdomen and pelvis to determine the extent of the tumor. CT chest, bone scan, bone marrow aspirate and biopsy were done for metastatic evaluation. Routine lymph node sampling was not required. Patients were staged according to the Intergroup Rhabdomyosarcoma Study Group (IRSG) pretreatment staging system and the IRSG surgico-pathologic group classification [12]. At diagnosis, patients were categorized into three risk groups (Low, intermediate, and high) according to the stage, clinical group and histological subtype which was derived from COG risk stratification system.

\subsection{Treatment}

Systemic treatment was given based on the risk group assignment, mainly vincristine, dactinomycin and cyclophosphamide (VAC) as the standard chemotherapy regimen. For the intermediate risk (IR) group the cyclophosphamide (CTX) was given in 14 cycles while it was in 4 cycles only for the low risk (LR) patients

\subsection{Local Control}

Surgery: An initial surgical resection was performed only if it was possible to achieve gross total resection without compromising bladder function. In most cases, a biopsy was the initial procedure. Delayed or planned surgical resection was done in patients below one year at diagnosis, or cases that showed poor response to chemotherapy and had bulky tumors at times of local control.

Radiotherapy: According to the treatment protocol, clinical group I patients received no further local therapy. Group II and group III patients received RT beginning either at/after week12 (from 2007 to 2010), or at/after week 4 (from 2011 till now). Patients with Group III tumors who underwent second-look operation (SLO) received dose adjusted RT within 2 weeks after surgical excision. Clinical group II patients received 36-41.4 Gy while it was 45-50.4 Gy for those with clinical group III disease. All patients underwent position fixation and immobilization using thermoplastic pelvic mask together with IV contrast during the process of CT simulation. CT images were taken every 2-3 $\mathrm{mm}$ and transferred to treatment planning system (TPS) for delineation. Gross tumor volume (GTV), Clinical tumor volume (CTV) was initiated by adding $11.5 \mathrm{~cm}$ margin to GTV using the initial pre-chemotherapy volume for the first phase and post chemotherapy volume for booster dose. PTV was formed by adding the margins to counteract the effect of position uncertainty, according to our department policy. Normal structures were also contoured including the rectum, penile bulb, testes, bowel (to the level of S1), femoral heads and pelvic growth plates. The radiotherapy planning was executed either using conformal plan (Xio planning system, CMS) or IMRT (Kon Radplanning system, Siemens). The best plan for better coverage of the tumor simultaneously with a maximum decrease of the radiation dose to the normal tissues was chosen for each individual patient.

\subsection{Statistical Methods}

Complete remission (CR) was defined as either the absence of any residual radiologic abnormalities, or the presence of residual abnormalities that remained stable for 6 months after the end of therapy. The outcome was defined both by overall survival (OS) and Failure-Free survival (FFS). OS was calculated from the time of diagnosis to death from any cause or last follow-up. FFS was calculated from the time of diagnosis to first relapse or progression, or last follow-up. OS and FFS were calculated using the Kaplan - Meier method. The statistical significance of each variable was tested by the log-rank test (Univariate procedure). Follow-up data were obtained through Jan 2018. SPSS was used for all statistical analysis (IBM Corp. Released 2013. IBM SPSS Statistics for Windows, Version 22.0. IBM Corp., Armonk, NY)

\section{Results}

The study included 54 patients with localized B-PRMS; all of them had embryonal histology. The median age at diagnosis was 3.28 years (range 4 months - 13.5 years) with $1: 4 \mathrm{M} / \mathrm{F}$ ratio, the Characteristics of the whole patients' cohort are shown in table 1 . The Tumor was involving the trigone and the 
bladder neck in 46 patients (85\%), while 8 patients had were diagnosed with clinical group III disease (initial biopsy bladder masses at the superior surface. Fifty one patients $(94 \%)$ only) and treated according to IR protocol.

Table 1. Patient characteristics.

\begin{tabular}{|c|c|c|}
\hline & Category $(n=54)$ & Frequency n (\%) \\
\hline \multirow[t]{2}{*}{ Age Median- range } & 3.28 (4 months-13.5 years) & \\
\hline & $\mathrm{a}=<5 \mathrm{~cm}$ & $22(41 \%)$ \\
\hline \multirow[t]{3}{*}{ Tumor size } & $\mathrm{b}>5 \mathrm{~cm}$ & $29(54 \%)$ \\
\hline & Unknown & $3(5 \%)$ \\
\hline & Confined: 31 (57\%) & \\
\hline \multirow[t]{3}{*}{ Tumor invasiveness } & Extensive: $17(31 \%)$ & \\
\hline & Unknown: $6(12 \%)$ & \\
\hline & Not clinically involved 42 (78\%) & \\
\hline \multirow[t]{2}{*}{ LN involvement } & Clinically involved: $10(18.5 \%)$ & \\
\hline & Unknown: $2(3.5 \%)$ & \\
\hline \multirow{3}{*}{ Gender } & Female & $44(81.5 \%)$ \\
\hline & Male & $10(18.5 \%)$ \\
\hline & Bladder base & $43(79.5 \%)$ \\
\hline \multirow[t]{2}{*}{ Exact location } & Bladder dome & $8(15 \%)$ \\
\hline & Prostate & $3(5.5 \%)$ \\
\hline \multirow{2}{*}{ Stage } & Stage 2 & $21(39 \%)$ \\
\hline & Stage 3 & $33(61 \%)$ \\
\hline \multirow{3}{*}{ Group } & Group II & $3(5.6 \%)$ \\
\hline & Group III & $51(94.4 \%)$ \\
\hline & Botryoid & $13(24 \%)$ \\
\hline \multirow[t]{2}{*}{ Pathology } & Spindle cell & $5(9 \%)$ \\
\hline & Embryonal, NOS & $36(67 \%)$ \\
\hline \multirow{2}{*}{ Surgery as LC } & Gross initial excision & 3 \\
\hline & Gross delayed excision (week 10) & 4 \\
\hline \multirow{4}{*}{ Method of LC } & Surgery only & $3(5.6 \%)$ \\
\hline & Gross excision surgery + Rth & $4(7.4 \%)$ \\
\hline & Rth only & $43(79.6 \%)$ \\
\hline & None & $4(7.4 \%)$ \\
\hline \multirow{2}{*}{ Time of Rth } & Early (before week 12) & $29(61.7 \%)$ \\
\hline & Delayed (at or after week 12) & $18(38.3 \%)$ \\
\hline
\end{tabular}

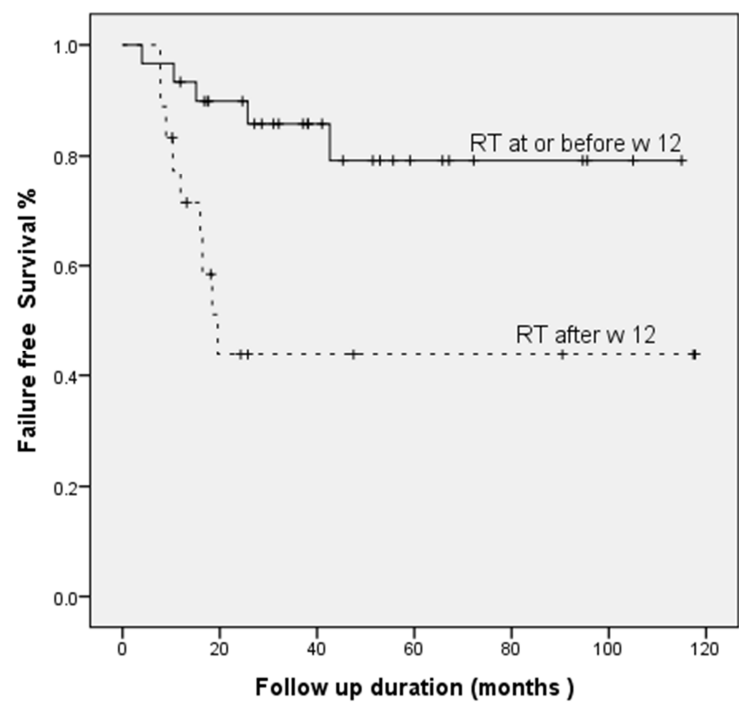

Figure 1. Failure free survival in relation to timing of RTH.

Of the whole patient cohort, 3 patients underwent initial partial cystectomy $(\mathrm{R} 0 \mathrm{n}=2, \mathrm{R} 1 \mathrm{n}=1)$ with primary closure, one of them had postoperative radiotherapy and none of these patients had disease relapse.

Delayed surgical resection after primary chemotherapy was done in 4 patients $(7.4 \%), 1$ patients had partial cystectomy with primary closure and 3 patients had partial cystectomy with ileocystoplasty. Three out of four patients received postoperative radiotherapy. Two of the 4 patients had disease relapse.

Radiotherapy as the only local treatment modality was used in 43 patients. The timing of local RTH was variable among our patients because of many factors like the treatment protocol (before or after

2010), patients age, 2nd look surgery and response to primary chemotherapy, so 30 patients received early RTH (before week 12) while 18 received delayed RTH (after week 12). Twelve out of the 43 patients had Disease relapse. Local control was not done in four patients, as they had poor response to treatment so they were given salvage chemotherapy but they had disease progression. With a median duration of follow up of 38.12 (8-112 months), the 5 -year failure-free survival (FFS) and overall (OS) of the whole patients were $60.4 \pm 14.5 \%$ and $75.4 \pm 14.1 \%$ respectively. The FFS and OS were significantly affected by the timing of local control (before or at week $12 \mathrm{Vs}$ after week 12). The FFS before and after week 12 was $79.2 \% \pm 17 \%$ and $43.8 \% \pm 25 \%$ respectively with $\mathrm{P}$ value 0.005 (Fig 1 ) and the OS before and after week 12 was $92.9 \% \pm 13.5 \%$ and $44.5 \% \pm$ $30.2 \%$ respectively with $\mathrm{P}$ - value 0.007 (fig 2). The OS was also significantly affected by tumor size $(\leq 5 \mathrm{~cm}$ was $95.5 \% \pm 8.6 \%$ and $>5 \mathrm{~cm}$ was $58.5 \% \pm 22.1 \%$ with P-value 
0.007). Other prognostic factors did not affect OS and FFS table 2 .

Nineteen patients $(35 \%)$ had disease relapse (local $n=17$ and distant $\mathrm{n}=2$ ). Out of the 17 patients with local relapse, 7 patients had salvage surgery: partial cystectomy and ileocystoplasty $n=1$, radical cystectomy and ileal loop conduit $\mathrm{n}=2$. In the other 4 patients, radical cystectomy and reconstruction using orthotopic continent urinary diversion (studer neobladder) was done.

The reported postoperative complications among the 11 patients who had surgery were a postoperative intestinal obstruction (one case), prolonged urinary leak (one case) and wound dehiscence in one case.

Table 2. Survival outcome according to different prognostic factors.

\begin{tabular}{|c|c|c|c|c|c|c|}
\hline Patient parameter & 5-year FFS & $95 \% \mathrm{CI}$ & P-value & 5-year OS & $95 \%$ CI & Pvalue \\
\hline \multicolumn{7}{|l|}{ Age } \\
\hline$\leq 1$ years & $36 \pm 31.5 \%$ & & 0.053 & $53.3 \% \pm 34.4 \%$ & & \\
\hline$>1$ years & $66.1 \pm 15.8 \%$ & & & $82.1 \% \pm 13.9 \%$ & & 0.235 \\
\hline \multicolumn{7}{|l|}{ Tumour invasiveness } \\
\hline Confined: & $73.9 \pm 17.24$ & & 0.334 & $90.9 \% \pm 12.5 \%$ & & 0.348 \\
\hline Extensive: & $55.5 \pm 25.7$ & & & $76.5 \pm 24.7$ & & \\
\hline \multicolumn{7}{|l|}{$\mathrm{LN}$ involvement } \\
\hline Not clinically involved: & $58.7 \%$ & & 0.518 & $74.6 \%$ & & 0.482 \\
\hline Clinically involved: & $51.4 \%$ & & & 76.2 & & \\
\hline \multicolumn{7}{|l|}{ Tumor size } \\
\hline$\leq 5 \mathrm{~cm}$ & $72.7 \pm 18.6 \%$ & & 0.252 & $95.5 \pm 8.6 \%$ & & \\
\hline$>5 \mathrm{~cm}$ & $45.9 \pm 21.4 \%$ & & & $58.5 \pm 22.1 \%$ & & 0.007 \\
\hline \multicolumn{7}{|l|}{ RTH Timing } \\
\hline Before or at week 10 & $79.2 \pm 17 \%$ & & 0.005 & $92.9 \% \pm 13.5$ & & \\
\hline After week 10 & $43.8 \pm 25 \%$ & & & $44.5 \pm 30.2 \%$ & & 0.007 \\
\hline
\end{tabular}

RTH; radiation therapy NA; Not Applicable as median was not reached

\section{Discussion}

Adequate treatment strategy for patients with bladder/prostate RMS requires maintaining the organ function by an understanding of the likely long-term complications of the given treatment, in addition to the achievement of adequate survival rates. Over the past several years, studies by the Intergroup Rhabdomyosarcoma Study Group (IRSG, the precursor to the COG) and SIOP focused on conservative approaches by use of chemotherapy and radiotherapy in order to avoid radical surgical excision and to maintain bladder function $[7,13,14]$. The choice of local control modality should be individualized based on patient age, tumor site \& size, response to chemotherapy and capability of conservative surgical excision. Although, non-mutilating surgery and LDR-brachytherapy has been advocated as successful treatment modality for BPRMS, the major problem of LDR-brachytherapy availability remains substantial $[15,16]$. In the present study we tried to evaluate the efficiency of our treatment strategy and to assess the local control problems that affect the outcome of these patients. One of the essential challenges in the local treatment of B/PRMS is the assessment of the feasibility of complete tumor resection without loss of organ function. In the present study, only 3 patients $(5.5 \%)$ had primary complete surgical resection ( $\mathrm{RO} n=2, \mathrm{R} 1 \mathrm{n}=1$ ) and 4 patients (7.4\%) had delayed resection after preoperative chemotherapy. The extent of bladder involvement determines the extent of resection which is a major factor in choosing the mode of reconstruction. In the 3 patients who underwent primary surgical resection, the lesion was domal and /or pedunculated which allowed initial surgical resection with primary closure. In the other 4 patients who received surgery at the time of local control, the tumor was away from the bladder trigon, which allows partial cystectomy with primary closure in 1 cases and partial cystectomy and ileocystoplasty in the other 3 cases. Among the 7 patients who underwent salvage surgery, 1 patient had partial cystectomy and ileocystoplasty as the tumor was away from the bladder trigon. Two patient had radical cystectomy and ileal loop conduit because the tumor was extending to urethra. In the other 4 patients, the lesion was extending to the bladder neck but not to the urethra, so radical cystectomy was performed and reconstruction using orthotopic continent urinary diversion (studer neobladder). Studies like CW96 reported higher operability rates as there were $11 \%$ of their patients underwent primary complete resection (R0 or R1), $19 \%$ with secondary tumor resection after preoperative chemotherapy and another $20 \%$ after preoperative radio chemotherapy [10]. But we should notify that the operability in the present study was largely affected by tumor site, size and invasiveness. Also the acceptable surgical approach in first-line treatment of these patients was only resection with primary closure. The main aim of the serial MMT trials 84 , 89 and 95 was to reduce the severity of the local treatment burden and they reported that conservative surgical excision either initial or after preoperative chemotherapy could be done in $58 \%$ of their patients [14]. The new trend of using preoperative radiotherapy that was addressed in CW96 to achieve further response before conservative adequate surgical excision was also advocated for the IRS-IV study [7]. The $(75.4 \pm 14.1 \%) 5$-year OS rate in the present cohort was not far from the other comparable international group studies. While the FFS $(60.4 \pm 14.5 \%)$ is lower than that reported by the IRS IV ( $75 \%$ at 3 years) which was giving the 
same chemotherapy regimen (VAC) with intensified cyclophosphamide [16, 17] and CWS 86, 96 studies (70\%5-year EFS) using VIA \pm doxorubicin and radiotherapy based on patient age and response to the neoadjuvant chemotherapy $[16,17,10]$. The SIOP reported $64 \% 5$ - year EFS in MMT 89 protocol with three drug regimen IVA which was not significantly improved when received six drugs (IVA, carboplatin, epirubicin, and etoposide) in MMT95 study (67\% at 5 years) $[9,18,19]$. On the same time OS was almost the same between all groups. So there was no significant difference between the previously known chemotherapeutic agents even with increasing their intensity. Newer agents may be required if we need to achieve a better chemotherapy response. Systemic chemotherapy in RMS has major role in disease control by prevention of metastatic disease and also for reduction of the primary tumor size in order to facilitate further local therapy [20, 21], but the number of chemotherapy cycles before the local control (surgery or/and RTH) should not be prolonged waiting for further response as this may be accompanied by impaired disease control. The present study showed significant improvement in FFS for patients who did early local control (before week 12) as compared to those who had postponed the time of local therapy (after week 12). This observation is consistent with what was indicated by the CWS-96study about the danger of delaying definitive local surgical therapy while waiting for chemotherapy and radiotherapy to take effect [10]. The rationale for early RT is the reduced local failure rate for patients with parameningeal tumors and intracranial extension who receive RT within 2 weeks of starting systemic therapy ( $18 \%$ vs. $33 \%$ for delay beyond 2 weeks). However, no statistical difference in FFS was seen by timing of RT, the question of RT timing was more directly evaluated at ARST0531 which aims to evaluate the benefit of earlier onset of RT (at week 4) [22]. The corner stone for the treatment success in this special group of patients is to make a balance between the efficiency of the treatment modality to control the disease and the cost of that modality as regard the organ function. While in case of relapse, radical surgical resection is essential for long term survival. In the present study 19 patients had relapsed (local $n=17$ and distant $n=2$ ), 7 of them were candidate for salvage resection; all of them were alive at the last follow up. In conclusion. Timing of local control and local radiotherapy is crucial and shouldn't be delayed waiting for further response to the systemic chemotherapy. Limitations of this study includes retrospective nature of the study, non uniform timing of radiation therapy and the FOXO1 fusion status was not available.

\section{Conclusions}

Timing of local control and local radiotherapy is crucial and shouldn't be delayed waiting for further response to the systemic chemotherapy.

All authors have no financial or personal relationships with other people or organizations that could inappropriately influence (bias) their work. We have no conflict of interest related to the content of this paper.

\section{References}

[1] Spunt SL, Sweeney TA, Hudson MM, Billups CA, Krasin MJ, Hester AL. Late effects of pelvic rhabdomyosarcoma and its treatment in female survivors. J Clin Oncol 2005; 23:7143-51. doi:10.1200/JCO.2005.12.096.

[2] Lott S, Lopez-Beltran A, Montironi R, MacLennan GT, Cheng L. Soft tissue tumors of the urinary bladder. Hum Pathol 2007; 38:963-77. doi:10.1016/j.humpath.2007.03.016.

[3] Hays DM, Raney RB, Wharam MD, Wiener E, Lobe TE, Andrassy RJ, et al. Children with vesical rhabdomyosarcoma (RMS) treated by partial cystectomy with neoadjuvant or adjuvant chemotherapy, with or without radiotherapy. A report from the Intergroup Rhabdomyosarcoma Study (IRS) Committee. J Pediatr Hematol Oncol 1995; 17:46-52.

[4] Pappo AS, Shapiro DN, Crist WM, Maurer HM. Biology and therapy of pediatric rhabdomyosarcoma. J Clin Oncol 1995; 13:2123-39. doi:10.1200/JCO.1995.13.8.2123.

[5] Meza JL, Anderson J, Pappo AS, Meyer WH, Children's Oncology Group. Analysis of Prognostic Factors in Patients With Nonmetastatic Rhabdomyosarcoma Treated on Intergroup Rhabdomyosarcoma Studies III and IV: The Children's Oncology Group. J Clin Oncol 2006; 24:3844-51. doi:10.1200/JCO.2005.05.3801.

[6] Hays DM. Bladder/prostate rhabdomyosarcoma: results of the multi-institutional trials of the Intergroup Rhabdomyosarcoma Study. Semin Surg Oncol n.d.; 9:520-3.

[7] Arndt C, Rodeberg D, Breitfeld PP, Raney RB, Ullrich F, Donaldson S. Does bladder preservation (as a surgical principle) lead to retaining bladder function in bladder/prostate rhabdomyosarcoma? Results from intergroup rhabdomyosarcoma study iv. J Urol 2004; 171:2396-403.

[8] Fryer CJ. Pelvic rhabdomyosarcoma: paying the price of bladder preservation. Lancet (London, England) 1995; 345:141-2.

[9] Stevens MCG, Rey A, Bouvet N, Ellershaw C, Flamant F, Habrand JL, et al. Treatment of nonmetastatic rhabdomyosarcoma in childhood and adolescence: third study of the International Society of Paediatric Oncology--SIOP Malignant Mesenchymal Tumor 89. J Clin Oncol 2005; 23:2618-28. doi:10.1200/JCO.2005.08.130.

[10] Seitz G, Dantonello TM, Int-Veen C, Blumenstock G, Godzinski $\mathrm{J}$, Klingebiel T, et al. Treatment efficiency, outcome and surgical treatment problems in patients suffering from localized embryonal bladder/prostate rhabdomyosarcoma: A report from the cooperative soft tissue sarcoma trial CWS-96. Pediatr Blood Cancer 2011; 56:718-24. doi:10.1002/pbc.22950.

[11] Ferrer FA, Isakoff M, Koyle MA. Bladder/prostate rhabdomyosarcoma: past, present and future. J Urol 2006; 176:1283-91. doi:10.1016/j.juro.2006.06.019.

[12] Malempati S, Hawkins DS. Rhabdomyosarcoma: review of the Children's Oncology Group (COG) Soft-Tissue Sarcoma Committee experience and rationale for current COG studies. Pediatr Blood Cancer 2012; 59:5-10. doi: $10.1002 /$ pbc. 24118 . 
[13] Stevens MCG. Treatment for childhood rhabdomyosarcoma: the cost of cure. Lancet Oncol 2005; 6:77-84. doi: 10.1016/S1470-2045(05)01733-X.

[14] Jenney M, Oberlin O, Audry G, Stevens MCG, Rey A, Merks JHM, et al. Conservative approach in localised rhabdomyosarcoma of the bladder and prostate: results from International Society of Paediatric Oncology (SIOP) studies: malignant mesenchymal tumour (MMT) 84,89 and 95 . Pediatr Blood Cancer 2014; 61:217-22. doi:10.1002/pbc.24727.

[15] Martelli H, Haie-Meder C, Branchereau S, Franchi-Abella S, Ghigna M-R, Dumas I, et al. Conservative surgery plus brachytherapy treatment for boys with prostate and/or bladder neck rhabdomyosarcoma: a single team experience. J Pediatr Surg 2009; 44:190-6. doi:10.1016/J.JPEDSURG.2008.10.040.

[16] Crist WM, Anderson JR, Meza JL, Fryer C, Raney RB, Ruymann FB, et al. Intergroup Rhabdomyosarcoma Study-IV: Results for Patients With Nonmetastatic Disease. J Clin Oncol 2001; 19:3091-102. doi:10.1200/JCO.2001.19.12.3091.

[17] Baker KS, Anderson JR, Link MP, Grier HE, Qualman SJ, Maurer HM, et al. Benefit of Intensified Therapy for Patients With Local or Regional Embryonal Rhabdomyosarcoma: Results From the Intergroup Rhabdomyosarcoma Study IV. J Clin Oncol 2000; 18:2427-34. doi:10.1200/JCO.2000.18.12.2427.

[18] Oberlin O, Rey A, Sanchez de Toledo J, Martelli H, Jenney
MEM, Scopinaro M, et al. Randomized Comparison of Intensified Six-Drug Versus Standard Three-Drug Chemotherapy for High-Risk Nonmetastatic Rhabdomyosarcoma and Other Chemotherapy-Sensitive Childhood Soft Tissue Sarcomas: Long-Term Results From the International Society of Pediatr. J Clin Oncol 2012; 30:245765. doi:10.1200/JCO.2011.40.3287.

[19] Jenney M, Oberlin O, Audry G, Stevens MCG, Rey A, Merks JHM, et al. No Title 2014; 61. doi:10.1002/pbc.24727.

[20] Raney B, Anderson J, Jenney M, Arndt C, Brecht I, Carli M, et al. Late effects in 164 patients with rhabdomyosarcoma of the bladder/prostate region: a report from the international workshop. J Urol 2006; 176:2190-4; discussion 2194-5. doi:10.1016/j.juro.2006.07.064.

[21] Modritz D, Ladenstein R, Pötschger U, Amman G, Dieckmann $\mathrm{K}$, Horcher E, et al. Treatment for soft tissue sarcoma in childhood and adolescence. Austrian results within the CWS 96 study. Wien Klin Wochenschr 2005; 117:196-209.

[22] Michalski JM, Meza J, Breneman JC, Wolden SL, Laurie F, Jodoin MA, et al. Influence of radiation therapy parameters on outcome in children treated with radiation therapy for localized parameningeal rhabdomyosarcoma in Intergroup Rhabdomyosarcoma Study Group trials II through IV. Int J

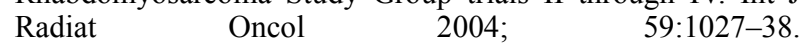
doi:10.1016/J.IJROBP.2004.02.064. 